\title{
Differential Localization of Chemotactic Signaling Arrays during the Lifecycle of Vibrio parahaemolyticus
}

\author{
Jan Heering and Simon Ringgaard* \\ Department of Ecophysiology, Max Planck Institute for Terrestrial Microbiology, Marburg, Germany
}

When encountering new environments or changes to their external milieu, bacteria use elaborate mechanisms to respond accordingly. Here, we describe how Vibrio parahaemolyticus coordinates two such mechanisms - differentiation and chemotaxis. $V$. parahaemolyticus differentiates between two distinct cell types: short rod-shaped swimmer cells and highly elongated swarmer cells. We show that the intracellular organization of chemotactic signaling arrays changes according to the differentiation state. In swimmer cells chemotaxis arrays are strictly polarly localized, but in swarmer cells arrays form both at the cell poles and at irregular intervals along the entire cell length. Furthermore, the formation of lateral arrays increases with cell length of swarmer cells. Occurrence of lateral signaling arrays is not simply a consequence of the elongated state of swarmer cells, but is instead differentiation state-specific. Moreover, our data suggest that swarmer cells employ two distinct mechanisms for localization of polar and lateral signaling arrays, respectively. Furthermore, cells show a distinct differentiation and localization pattern of chemosensory arrays, depending on their location within swarm colonies, which likely allows for the organism to simultaneously swarm across surfaces while sustaining a pool of swimmers immediately capable of exploring new liquid surroundings.

*Correspondence: Simon Ringgaard simon.ringgaard@mpimarburg.mpg.de

Specialty section:

This article was submitted to Microbial Physiology and Metabolism, a section of the journal

Frontiers in Microbiology

Received: 19 September 2016

Accepted: 21 October 2016

Published: 02 November 2016

Citation:

Heering J and Ringgaard S (2016)

Differential Localization

of Chemotactic Signaling

Arrays during the Lifecycle of Vibrio

parahaemolyticus.

Front. Microbiol. 7:1767.

doi: 10.3389/fmicb.2016.01767

\section{INTRODUCTION}

Bacteria often experience changes in their external environment and have developed various strategies to respond accordingly. One mechanism to accommodate such changes involves the differentiation into specialized cell types suitable for the particular conditions. Differentiation often involves major changes in the cell cycle, cell morphology, and the spatiotemporal organization of cells. A distinct type of differentiation utilized by many bacteria, including species of Serratia (Alberti and Harshey, 1990), Aeromonas (Kirov et al., 2002), Salmonella (Harshey, 1994; Harshey and Matsuyama, 1994), Proteus (Rather, 2005), and Vibrio (McCarter, 2004), is the differentiation between a planktonic swimmer cell and a swarmer cell that is specialized for movement over solid surfaces or in viscous environments (McCarter, 2004). One organism that undergoes such differentiation between swimmer and swarmer cells is Vibrio parahaemolyticus, a worldwide human pathogen and major cause of seafood related gastroenteritis (McCarter and Silverman, 1990; McCarter, 1999, 2004, 2010; Makino et al., 2003; Stewart and McCarter, 2003; Gode-Potratz and McCarter, 2011). In V. parahaemolyticus swimmer cells 
are short rod-shaped cells that - as the name suggests - are optimized for swimming in liquid environments. However, when they encounter a solid surface, differentiation into a swarmer cell is triggered. Swarmer cells exist within bacterial communities of swarm colonies where they spread over surfaces. Within swarm colonies, there are differences in cell size - and likely also celltype - according to the position of cells within a swarm colony (Belas and Colwell, 1982; Roth et al., 2013). In the periphery of the swarm colony, cells assemble into flares that extend outward from the colony and cells stacked in a few layers. Closer to the center of the swarm colony cells are stacked in multiple layers and are considerably shorter than cells in the flares. Swarmer cells can maintain the swarmer lifestyle, where division events result in two new swarmer cells; alternatively, swarmers can de-differentiate back into swimmer cells, depending on the conditions (Figure 1). One of the first steps in swarmer differentiation is inhibition of cell division, resulting in highly elongated rod-shaped filamentous swarmer cells. A second major change during swarmer differentiation is the production of a multitude of lateral flagella, which are important for swarming behavior and likely used for surface contact, cell-cell contact, and interaction between groups of cells in order to coordinate their movement across surfaces (Baumann and Baumann, 1977; McCarter, 2004; Böttcher et al., 2016). Interestingly, the two flagellar systems used by swimmer and swarmer cells are distinct, but both appear to share the central chemotaxis system that is required for regulating chemotactic behavior and flagellar rotation (Sar et al., 1990).

Chemotaxis is an essential process for many motile bacteria to compete when encountering changes in their external milieu, and is one of the principal ways motile bacteria sense, respond, and adapt to changing environmental conditions. The process enables the bacteria to bias their movement away from unfavorable conditions and toward a more favorable external milieu (Wadhams and Armitage, 2004; Sourjik and

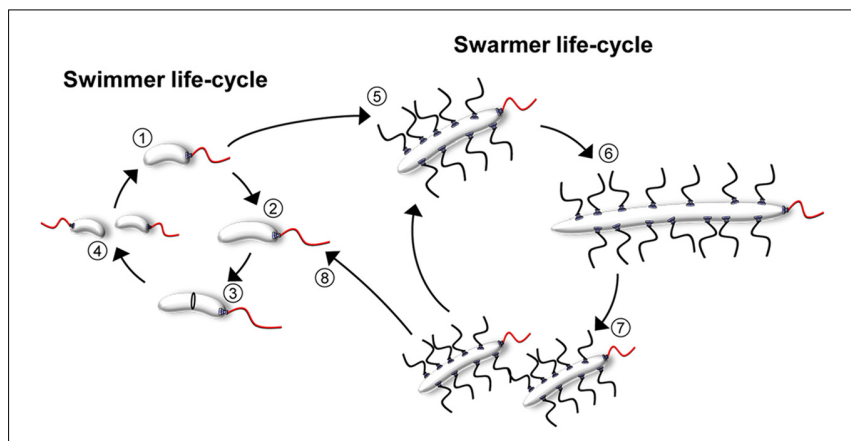

FIGURE 1 | The cell cycles of $\boldsymbol{V}$. parahaemolyticus. Schematic showing the life-styles and cell-cycles of $V$. parahaemolyticus. During the swimmer state, cells elongate (1-3) and eventually divide at mid-cell resulting in two progeny swimmer cells (4). Upon surface contact, swimmer cells can differentiate into the filamentous peritrichously flagellated swarmer cells (5). Swarmer cells can either continue the swarmer life-style, where division events result in swarmer progeny cells (5-7); alternatively swarmers can de-differentiate back into swimmer cells (8) and re-enter the swimmer cell cycle (1-4).
Armitage, 2010). Chemotaxis is mediated by large multicomponent clusters of signaling proteins, usually referred to as chemotactic signaling arrays. Chemotactic stimuli in the environment such as repellants or attractants are detected by chemosensory receptors termed "methyl-accepting chemotaxis proteins" (MCPs) at the cell surface. These receptors generally span the cytoplasmic membrane and interact in the cytoplasm with a histidine kinase, CheA. This interaction is stabilized by the cytoplasmic adaptor protein, CheW. If the signal perceived by the MCPs represents unfavorable environmental conditions, a phosphosignaling cascade via the histidine kinase CheA and the response regulator $\mathrm{CheY}$ is induced. Elevated levels of phosphorylated CheY increase the chance of a change in flagellar rotation and in consequence the direction of bacterial swimming - over time this results in a net movement toward more favorable conditions (Wadhams and Armitage, 2004; Sourjik and Armitage, 2010).

Chemotaxis has been extensively studied in the peritrichously flagellated bacterium Escherichia coli. In E. coli, array formation is believed to be a stochastic process (Thiem and Sourjik, 2008), resulting in localization of signaling arrays at the cell poles and non-regularly along the cell length (Sourjik and Berg, 2000). This pattern likely ensures that sensory arrays are localized in close proximity to the lateral flagella and that signaling arrays are stably inherited to the daughter cells at cell division. In other organisms, such as Caulobacter crescentus, Pseudomonas aeruginosa, Rhodobacter sphaeroides, and V. parahaemolyticus, chemosensory arrays are specifically localized at the cell poles (Alley et al., 1992; Maddock and Shapiro, 1993; Gestwicki et al., 2000; Wadhams et al., 2003; Bardy and Maddock, 2005). In particular, it was recently reported that chemotaxis arrays are exclusively directed to one or both cell poles in the polarly flagellated pathogens $V$. cholerae and $V$. parahaemolyticus by a novel mechanism (Ringgaard et al., 2011, 2014; Yamaichi et al., 2012). Here, the signaling arrays localize to the old flagellated cell pole immediately after cell division. Later in the cell cycle, the chemotaxis proteins are recruited to the new cell pole as the rod-shaped cell elongates, thus resulting in a bi-polar localization pattern; no lateral arrays are formed. The next cell division event then results in two daughter cells with one polar signal array each. It was recently discovered that proper polar localization and inheritance of signaling arrays depends on the ParA-like ATPase ParC (Ringgaard et al., 2011, 2014). In the absence of ParC, chemotaxis proteins are no longer recruited to the cell poles correctly. Instead, signaling arrays form and localize randomly along the cell length. As a consequence, bi-polar localization is not established prior to cell division and both daughter cells do not inherit a signaling array upon cell division. Mislocalization and unsuccessful segregation of signaling arrays to daughter cells result in altered motility and decreased chemotaxis (Ringgaard et al., 2011, 2014). Interestingly, fluorescence microscopy studies have suggested that changes occur in the localization of signaling arrays during differentiation of $V$. parahaemolyticus and that signaling arrays do not only localize to the cell poles in swarmer cells but also along the length of the cell (Gestwicki et al., 2000).

Here, we performed an in-depth analysis of the localization of chemotactic signaling arrays in $V$. parahaemolyticus during its 
differentiation cycle and within swarm colonies. In contrast to swimmer cells, signaling arrays are not exclusively localized to the cell poles in swarmer cells, but also form distinct clusters that localize along the cell length. Interestingly, we show that there is a correlation between swarmer cell length and the number of signaling arrays formed within the swarmer cell, where the number of lateral clusters formed increases with increased cell length. Moreover, lateral arrays do not localize in regular intervals along the cells length but are distributed irregularly along the entire length of the swarmer cell, and on average each cell halve hold the same number of arrays. Our data suggest that this localization pattern is not a consequence of cell elongation per $s e$, but instead formation of lateral sensory clusters is specific to the differentiated state of swarmer cells.

\section{MATERIALS AND METHODS}

\section{Growth Conditions and Media}

If not otherwise stated $E$. coli and $V$. parahaemolyticus were grown in LB media or on LB agar plates at $30^{\circ} \mathrm{C}$ or $37^{\circ} \mathrm{C}$ containing antibiotics in the following concentrations: streptomycin $200 \mu \mathrm{g} / \mathrm{ml}$; kanamycin $50 \mu \mathrm{g} / \mathrm{ml}$; ampicillin $100 \mu \mathrm{g} / \mathrm{ml}$; chloramphenicol $20 \mu \mathrm{g} / \mathrm{ml}$ for E. coli and $5 \mu \mathrm{g} / \mathrm{ml}$ for $V$. parahaemolyticus. When needed, L-arabinose was added to a final concentration of $0.2 \% \mathrm{w} / \mathrm{v}$.

\section{Strains and Plasmids}

The wild-type strain of $V$. parahaemolyticus used was the clinical isolate RIMD 2210633 and all mutants are derivatives of this strain. Strains and plasmids used throughout this study are listed in Table 1. Primers are listed in Table 2. E. coli strain $\mathrm{DH} 5 \alpha \lambda$ pir was used for standard cloning and SM10 $\lambda$ pir was used for transfer of plasmid DNA by conjugation from $E$. coli to $V$. parahaemolyticus. Deletion of genes in $V$. parahaemolyticus was performed using standard allele exchange techniques using derivatives of suicide vector pDM4 (Milton et al., 1996).

\section{Construction of Plasmids Plasmid pJH002}

The up- and down-stream regions flanking vpa1538 were amplified using primer pairs 146/63 and 64/147, respectively, using V. parahaemolyticus RIMD 2210633 chromosomal DNA as template. In a third PCR, using primers 146/147 and products of the first two PCR reactions as template, the flanking regions were stitched together. The resulting product was digested with XbaI and was inserted into the equivalent site of pDM4, resulting in plasmid pJH002.

\section{Plasmid pJH003}

The up- and down-stream regions flanking vpa1548 were amplified using primer pairs 148/68 and 69/149, respectively, using $V$. parahaemolyticus RIMD 2210633 chromosomal DNA as template. In a third PCR, using primers $148 / 149$ and products of the first two PCR reactions as template, the flanking regions were stitched together. The resulting product was digested with $\mathrm{XbaI}$ and was inserted into the equivalent site of $\mathrm{pDM} 4$, resulting in plasmid pJH003.

\section{Microscopy}

Fluorescence microscopy of swarming $V$. parahaemolyticus cells was carried out in several steps; $5 \mathrm{~mL}$ LB supplemented with the required antibiotic was inoculated with a colony of cells harboring the relevant plasmid and grown to $\mathrm{OD} 600=0.1$ at $37^{\circ} \mathrm{C}$ and shaking. Expression of fluorescent fusion proteins was induced by adding L-arabinose to a final concentration of $0.2 \%$ $\mathrm{w} / \mathrm{v}$. The cultures were incubated for an additional $2 \mathrm{~h}$ before $1.5 \mu \mathrm{L}$ were spotted in the center of a swarming agar plates, which subsequently were sealed with scotch-tape. Swarming agar plates were prepared from 40 g/L "Difco Heart Infusion Agar" (BD) supplemented with the required antibiotic, $4 \mathrm{mM} \mathrm{CaCl}^{2}$, $50 \mu \mathrm{M} \mathrm{2,2}$ '-Bipyridyl (Sigma-Aldrich), and 0.2\% L-arabinose. The sealed plates were then incubated for $16-18 \mathrm{~h}$ at $24^{\circ} \mathrm{C}$ to induce swarming. For microscopy, a piece of swarming agar containing cells was cut out from the plate and mounted onto agarose pads ( $1 \%$ agarose $\mathrm{w} / \mathrm{v}, 20 \% \mathrm{v} / \mathrm{v}$ PBS, and $20 \% \mathrm{v} / \mathrm{v} \mathrm{LB}$ ) on microscope slides.

In order to acquire stereomicroscopy images of swarming colonies, swarming plates were prepared as described, sealed and incubated for $16-18 \mathrm{~h}$ at $24^{\circ} \mathrm{C}$. Before acquiring the images, scotch tape and the lid of the swarming plate was removed. Stereomicroscopy was carried out using a Leica M205 FA Stereomicroscope.

Preparation of $V$. parahaemolyticus swimmer cells for fluorescence microscopy was carried out essentially as described (Ringgaard et al., 2015; Briegel et al., 2016). A volume of $10 \mathrm{~mL}$ of LB was inoculated with a bacterial colony of $V$. parahaemolyticus from an over-night LB agar plate grown at $37^{\circ} \mathrm{C}$ and relevant antibiotic. Cells were incubated for $1 \mathrm{~h}$ at $37^{\circ} \mathrm{C}$ and shaking after which $0.2 \% \mathrm{w} / \mathrm{v}$ L-arabinose was added in order to induce expression of YFP-CheW. Cells were then incubated for additionally $2 \mathrm{~h}$ and subsequently mounted on an agarose pad and imaged using fluorescence microscopy. For Aztreonam treatment, Aztreonam (Fluka) was added to a final concentration of $30 \mu \mathrm{g} / \mathrm{mL}$ after the $2 \mathrm{~h}$ of induction with $0.2 \% \mathrm{w} / \mathrm{v}$ L-arabinose. Cells were then incubated for additional $30 \mathrm{~min}$, mounted on an agarose pad and imaged by fluorescence microscopy. Fluorescence microscopy was carried out using a Nikon eclipse Ti inverted Andor spinning-disk confocal microscope equipped with a $100 \times$ lens and an Andor Zyla sCMOS cooled camera.

\section{Microscopy Image Analysis}

Before analysis, images generated by Nikon NIS-Elements AR were split up in single channels using Fiji/ImageJ 1.49j10 and each channel was saved as a separate tiff image. DIC and the corresponding fluorescent channel were loaded in MetaMorph Offline (version 7.7.5.0, Molecular Devices) where manual image analysis was performed. An overlay of both channels was generated before cells were marked using the "Multiline tool." The regions were then transferred to the original fluorescent channel image to extract the distances of foci and their distribution in $V$. parahaemolyticus cells. Using the linescan histogram, foci positions could be marked and copied to an 
TABLE 1 | Strains and plasmids list.

\begin{tabular}{|c|c|c|}
\hline Strain name & Genotype & Reference \\
\hline Vibrio parahaemolyticus RIMD 2210633 & Clinical isolate & Makino et al., 2003 \\
\hline V. parahaemolyticus MZ01 & RIMD $2210633 \Delta v p 2227$ parC & Ringgaard et al., 2014 \\
\hline V. parahaemolyticus SR58 & RIMD $2210633 \Delta v p 2225 \mathrm{cheW}$ & Ringgaard et al., 2014 \\
\hline V. parahaemolyticus JH2 & RIMD 2210633 svpa1548 lafA & This work \\
\hline V. parahaemolyticus JH5 & RIMD 2210633 svpa1538 lafk & This work \\
\hline Escherichia coli SM10גpir & $\mathrm{KmR}$, thi-1, thr, leu, tonA, lacY, supE, recA::RP4-2-Tc::Mu, $\lambda$ pir & \\
\hline E. coli DH5 $\alpha \lambda$ pir & 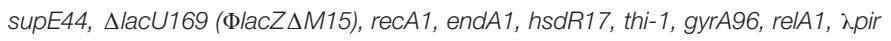 & \\
\hline Plasmid name & Relevant genotype/description & Reference \\
\hline pMZ03 & PBAD::yfp-vp2225 (cheW) & Ringgaard et al., 2014 \\
\hline pDM4 & Suicide vector for construction of deletion mutants; sacBR; oriR6K; $\mathrm{Cm}^{\mathrm{R}}$ & Milton et al., 1996 \\
\hline pJH002 & Plasmid for deletion of lafK & This work \\
\hline pJH0O3 & Plasmid for deletion of lafA & This work \\
\hline
\end{tabular}

TABLE 2 | Primers list.

\begin{tabular}{ll}
\hline Primer number & Primer sequence \\
\hline 63 & tcgtcatcattgaaccttaaccttc \\
64 & gaaggttaaggttcaatgatgacgacggtattgatttacagtcggct \\
68 & ataaagccatcttagtctccttag \\
69 & ctaaggagactaagatggctttatggcaatgtctctacttcgttaata \\
146 & cccctctagatctcgtcgatttgtattccgtaaag \\
147 & cccctctagaactctctaagaccgagacaatc \\
148 & cccctctagatgagcgtattgctgaatttgatcc \\
149 & cccctctagattatgtgttccgccttcctctc \\
\hline
\end{tabular}

Excel spreadsheet where further analysis and calculations were performed.

\section{Demographic Analysis of Microscopy Data}

Demographic analysis was performed in several steps: first fluorescence intensity profiles of cells were measured in Fiji/ImageJ, version 1.49j10. Afterward the generated data was processed in $\mathrm{R}$ [version 3.0.1; ( $\mathrm{R}$ Development Core Team, 2008)] with a script that sorts cells by length and normalizes the generated intensity profiles as an average of each cell's fluorescence. In $\mathrm{R}$ the ggplot2 package [version 1.0.0; (Wickham, 2009)] was used to produce the demographics.

\section{Electroporation of Plasmid DNA into V. parahaemolyticus}

Electrical-competent $V$. parahaemolyticus cells were produced by inoculating a single colony from a fresh agar plate in $200 \mathrm{ml}$ of $\mathrm{LB}$ medium and incubation at $37^{\circ} \mathrm{C}$ until an $\mathrm{OD}_{600}$ of 1.0 was reached. The cells were transferred onto ice immediately and all further steps were performed on ice and in pre-cooled centrifuges. The cells were harvested at $4^{\circ} \mathrm{C}$ for $10 \mathrm{~min}$ at $3500 \mathrm{rpm}$. After that, the supernatant was poured off and the cell pellet was re-suspended in $25 \mathrm{ml}$ of ice-cold $273 \mathrm{mM}$ sucrose solution ( $\mathrm{pH} 7.4$, buffered with $\mathrm{KOH}$ ). The cells were again harvested at $4^{\circ} \mathrm{C}$ for $10 \mathrm{~min}$ at $3500 \mathrm{rpm}$. This washing step was performed twice. Afterward the washed cell pellet was resuspended in $400 \mu \mathrm{l}$ of ice-cold $273 \mathrm{mM}$ sucrose solution with the addition of $15 \%$ glycerol. For the transformation, a $70 \mu \mathrm{l}$ aliquot of electrocompetent cells was mixed with 100-1000 ng of plasmid DNA and transferred into an ice-cold electroporation cuvette (Gene Pulser ${ }^{\circledR}$ Cuvette) and electroporated using the "GenePulser X-Cell" by BioRad with the following calibration: $25 \mu \mathrm{F}, 2400 \mathrm{~V}$ and $200 \Omega$. Afterward the cells were incubated shaking in $600 \mu \mathrm{l} \mathrm{LB}$ medium at $37^{\circ} \mathrm{C}$ for $1-3 \mathrm{~h}$. Later, the cultures where plated on selective $\mathrm{LB}$ agar plates and incubated at $37^{\circ} \mathrm{C}$ overnight.

\section{RESULTS}

\section{The Intracellular Organization of Chemosensory Arrays Changes during Differentiation of $V$. parahaemolyticus}

In order to investigate the intracellular localization and organization of chemosensory arrays during differentiation of $V$. parahaemolyticus, we ectopically expressed a fluorescently tagged version of CheW (YFP-CheW) in swimmer and swarmer cells. Despite YFP-CheW not being able to fully complement a strain lacking CheW, we have previously shown it can be used as a marker for signaling array localization in presence of wild type CheW (Ringgaard et al., 2014). In swimmer cells, YFPCheW localized in a uni- or bi-polar manner (Figures 2A-C) as previously reported: short cells displayed uni-polar localization whilst longer cells possessed bi-polar localization of signaling arrays (Ringgaard et al., 2014). Approximately 30\% of cells had uni-polar localization whilst $70 \%$ of cells showed a bi-polar localization pattern (Figure 3A). Interestingly, the intracellular localization of signaling arrays was different for swarmer cells. In almost $100 \%$ of swarmer cells YFP-CheW was found at both cell poles (Figure 3B); importantly, the localization of YFP-CheW clusters was not restricted to cell poles. Instead, clusters of YFPCheW could be observed localizing along the length of the cell (Figures 2D-F) in 55\% of cells (Figure 3B). Thus, in contrast to swimmer cells, a large proportion of swarmer cells form and localize chemotactic signaling arrays along the length of the cell. 
Localization of YFP-CheW in wild-type cells of V. parahaemolyticus
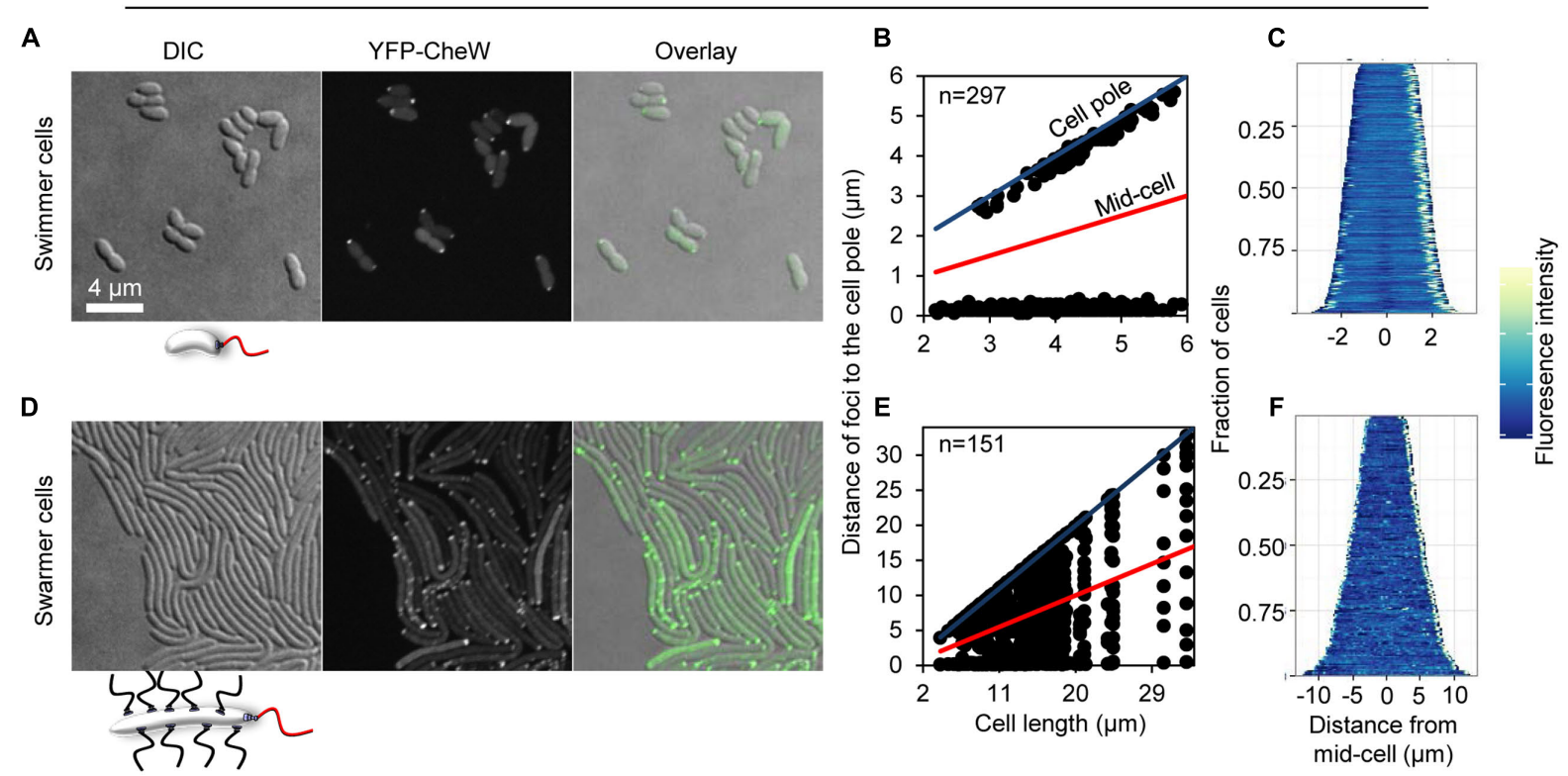

FIGURE 2 | Intracellular localization of chemotactic signaling arrays in swimmer and swarmer cells of $\boldsymbol{V}$. parahaemolyticus. Intracellular localization of YFP-CheW in wild-type swimmer (A-C) and swarmer cells (D-F) of V. parahaemolyticus. (A,D) Microscopy showing the intracellular localization of YFP-CheW in swimmer (A) and swarmer cells (D). (B,E) Graph depicting the distance of YFP-CheW foci from the cell poles as a function of cell length in swimmer (B) and swarmer cells (E). (C,F) demographic analysis showing the fluorescence intensity of YFP-CheW along the cell length in a population of $V$. parahaemolyticus relative to cell length in swimmer (C) and swarmer cells (F).

\section{Lateral Signaling Arrays are Distributed Through-Out the Length of the Cell in a Non-regular Manner}

In order to determine if there was a correlation between cell length and the number of lateral signaling arrays forming along the length of the cell in swarmer cells, we measured the number of YFP-CheW clusters as a function of cell length (Figure 4A). Interestingly, the number of lateral YFP-CheW clusters increased with increasing cell length. Thus, as swarmers elongate, there is a concomitant increase in the number of lateral signaling arrays.

Despite the non-regularity in array localization (Figures 2E,F), we analyzed if signaling arrays were distributed throughout the length of the cell. In order to do so, we calculated the percentile distribution of YFP-CheW clusters within specific regions of the cell (Figure 4B). On average each cell half possessed approximately $50 \%$ of signaling arrays, with $17 \%$ of arrays localizing within the mid-cell region (mid-cell $\pm 10 \%$ of cell length) (Figure 4B). Thus, signaling arrays are distributed along the entire length of the swarmer cell and on a population average each cell half contains the same number of signaling arrays.

\section{Lateral Localization of Signaling Arrays in Swarmer Cells Is Not a Function of Cell Length but Specific to the Differentiation State}

We wanted to investigate if lateral signaling arrays also form in elongated cells from liquid media that have not initiated swarmer differentiation. Thus, we analyzed array localization in swimmer cells treated with the cell division inhibitor beta-lactam antibiotic Aztreonam. Aztreonam inhibits the function of the cell division protein FtsI, which results in the formation of elongated swimmer cells comparable in cell length to that of swarmer cells. Under these conditions, chemotactic signaling arrays were always localized at the cells poles (4\% uni-polar and 96\% bi-polar localization) and as regular bands corresponding to midcell or quarter-cell positions in 70\% of cells (Figures 3A and 5), suggesting an association of YFP-CheW with the cell division apparatus. This implies that lateral localization of signaling arrays along the cell length observed in swarmer cells is not simply a consequence of the elongated state of swarmer cells, but is in fact due to specific changes in the intracellular organization and localization of signaling arrays during differentiation between swimmer and swarmer cells.

\section{ParC Is Required for Polar Localization of Signaling Arrays in Swimmer and in Swarmer Cells}

We have previously shown that the ParC-system is responsible for polar localization of signaling arrays in swimmer cells. In order to determine if ParC also directs localization of signaling arrays in swarmer cells, we analyzed the localization in YFP$\mathrm{CheW}$ in a strain deleted for parC and compared it to wild-type. As expected, signaling arrays were no longer recruited to the cell poles in the absence of ParC in swimmer cells, but formed clusters 

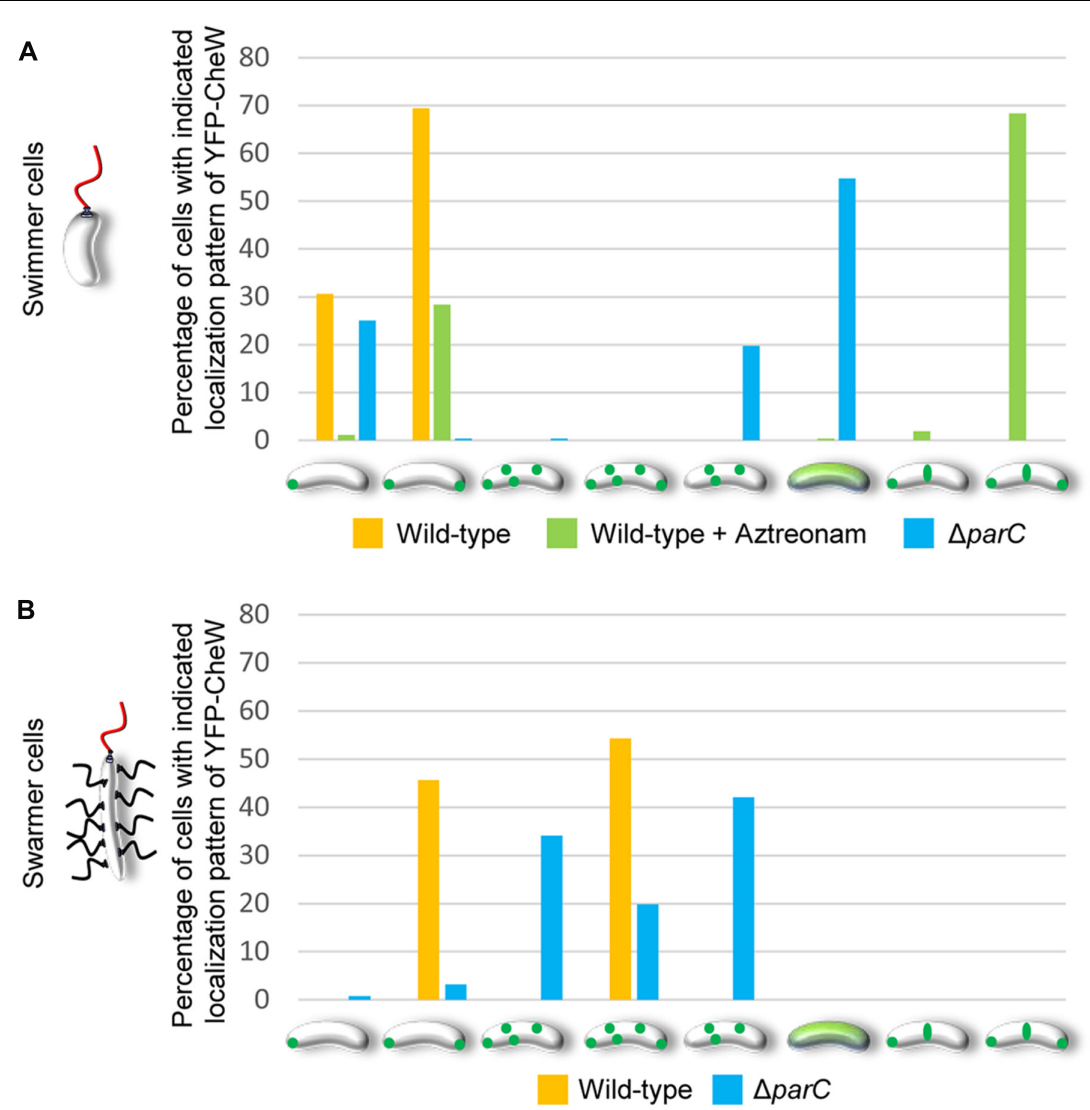

FIGURE 3 | Changes in the intracellular localization of chemotactic signaling arrays during differentiation of $\boldsymbol{V}$. parahaemolyticus. Localization pattern of YFP-CheW in swimmer (A) and swarmer cells (B) of wild-type, Aztreonam treated swimmer cells, and $\Delta$ parC strains of $V$. parahaemolyticus. Bar graphs show the percentage of cells with the indicated distinct localization patterns. (A) Number of cells analyzed, $n$ : wild-type, $n=298$; wild-type + Aztreonam, $n=272 ; \Delta p a r C$, $n=320$. (B) Number of cells analyzed, $n$ : wild-type, $n=151 ; \Delta$ parC, $n=126$.

at random positions along the cell length. Consequently daughter cells did not faithfully inherit an array at cell division (Figures $\mathbf{3 A}$ and 6A-C) (Ringgaard et al., 2011, 2014). Strikingly, in swarmer cells lacking ParC (Figures 6D-F) merely 23\% of cells showed a bi-polar localization pattern of YFP-CheW (Figure 3B). Instead, YFP-CheW was localized uni-polarly in $45 \%$ of cells and $33 \%$ of cells completely lacked polar YFP-CheW clusters (Figure 3B). Thus, approximately $77 \%$ of swarmer cells failed to establish bi-polar localization in the absence of ParC (Figures 3B and 6DF). These data show that ParC is required for polar recruitment of YFP-CheW and chemotactic signaling arrays to the cell poles in both swimmer and swarmer cells.

\section{Formation and Localization of Lateral Signaling Arrays in Swarmer Cells Is Independent of ParC}

Interestingly, chemotactic sensory arrays still formed and localized along the length of the cell in swarmer cells in absence of ParC. In order to analyze if the distribution of lateral signaling arrays was dependent on ParC, we measured the average distance between adjacent clusters of YFP-CheW relative to cell length and the number of YFP-CheW clusters per cell in wild-type and $\triangle$ parC backgrounds (Figure 6G). As expected the average distance between clusters of YFP-CheW decreased as the number of clusters increased. Interestingly, the average distance between clusters was indistinguishably between wild-type and cells lacking ParC ( $p=0.387$ ) (Figure 6G), hence indicating that localization and spacing between lateral signaling arrays is independent of the ParC-system and is guided by a different mechanism.

\section{ParC Is Required for Optimal Swarming Behavior}

Since ParC is required for establishing bi-polar localization of chemotactic signaling arrays in swarmer cells, we investigated if ParC was required for swarming behavior of $V$. parahaemolyticus. To this end, we performed swarming assays with strains lacking ParC and compared it to wild-type cells. As negative controls for swarming, we included strains lacking the major lateral flagellin LafA or the swarmer specific sigma-factor LafK. Additionally, we included a strain lacking the chemotaxis protein $\mathrm{CheW}$ that does not show chemotactic behavior of swimmer cells (Ringgaard et al., 2014). As expected, wild-type cells formed large swarm colonies when spotted on swarm-agar plates and no swarming was observed for cells lacking LafA or LafK (Figures 6H,I). 


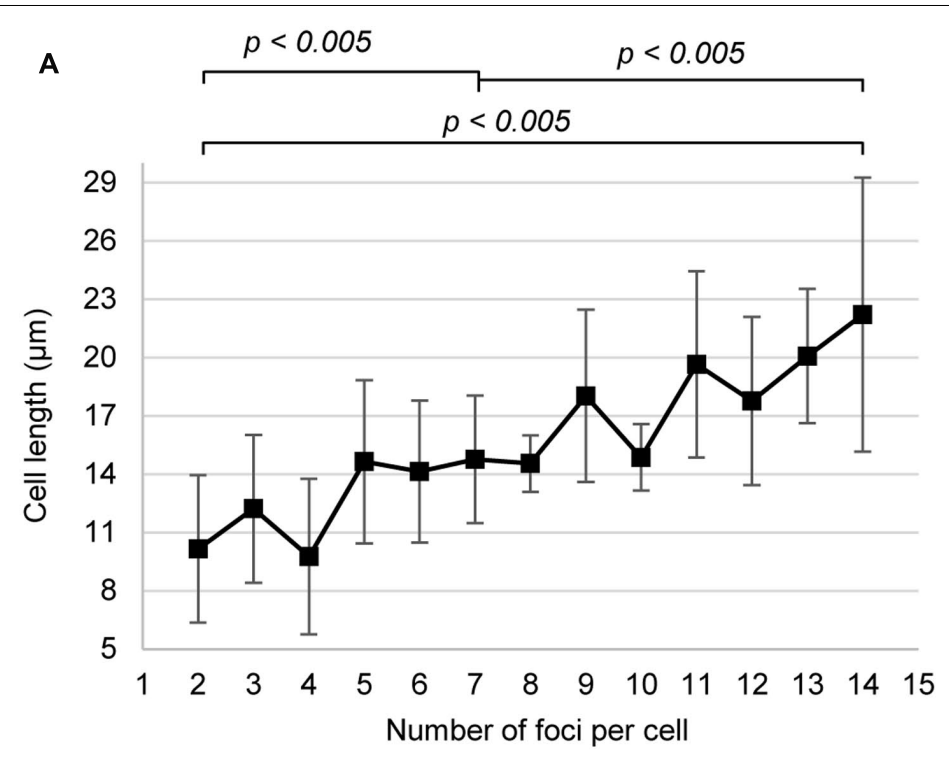

B

The percentile distribution of YFP-CheW clusters within regions of $V$. parahaemolyticus swarmer cells

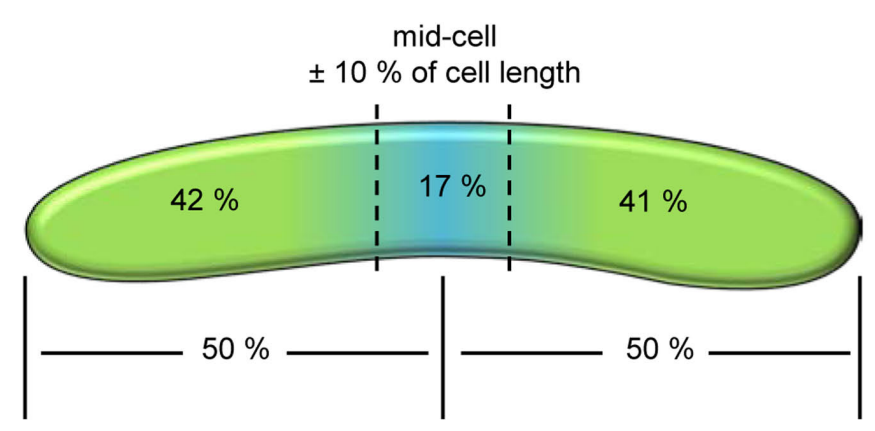

FIGURE 4 | A cell length dependent, non-regular distribution of lateral signaling arrays through-out the entire length of swarmer cells. (A) Graph depicting the number of YFP-CheW clusters as a function of cell length. (B) Schematic showing the percentile distribution of YFP-CheW clusters within regions of V. parahaemolyticus swarmer cells.

A strain lacking CheW showed an $80 \%$ reduction in swarming compared to wild-type, showing that the chemotactic system is required for proper swarming behavior. Interestingly, cells lacking ParC also showed a reduction in swarming colony formation of almost 50\% compared to wild-type (Figures 6H,I). Thus, ParC plays a role in swarming of $V$. parahaemolyticus, suggesting that swarmer cells require a bi-polar localization of chemotactic signaling arrays in order to swarm in an optimal manner.

\section{Swarm-Colonies Consist of Different Cell Types with Distinct Localization Patterns of Chemotactic Signaling Arrays}

There are major differences in cell size - and likely also celltype - according to the position of cells within a swarm colony [(Belas and Colwell, 1982), Figure 7]. In the periphery of the swarm colony, cells assemble into flares that extend outward from the colony. The flares consist of cells stacked in a few layers, thinning to a monolayer of long swarmer cells at the tip of the flares (Figures 7A,C). By contrast, closer to the center of the swarm colony cells are stacked in multiple layers and are considerably shorter than cells in the flares and more resembling stationary phase swimmer cells in cell length (Figures 7A,C). Due to the differences in internal organization of signaling arrays between swimmer and long swarmer cells, we hypothesized that $V$. parahaemolyticus also regulates its internal organization of signaling arrays depending on the cells position within swarm colonies. Thus, we analyzed the localization of YFP-CheW in cells originating from swarm flares and from the middle of the swarm colony (Figure 7B). In contrast to the long swarmer cells in the swarm-flares, in the vast majority of cells from the center of a swarm colony YFP-CheW localized to the cell poles in a uni- and bi-polar manner and only rarely was a lateral YFP-CheW cluster 


\section{Localization of YFP-CheW in artificially elongated (Aztreonam) wild-type swimmer cells of $V$. parahaemolyticus}

A

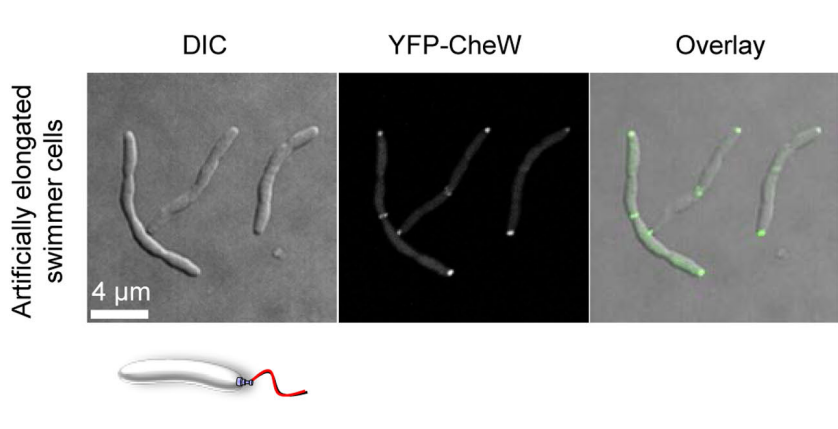

B

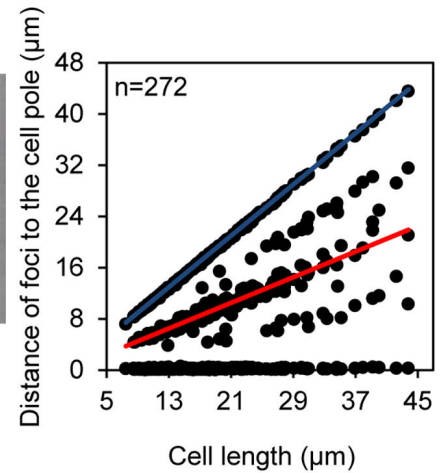

C

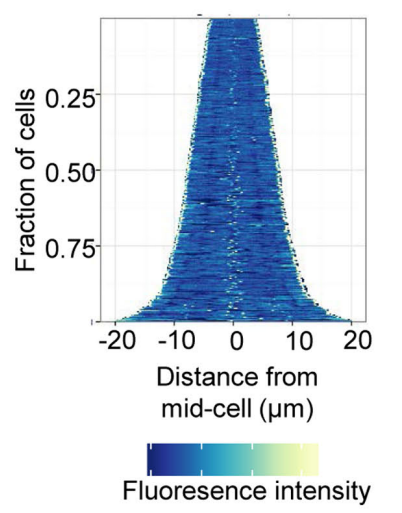

FIGURE 5 | The intracellular formation of chemotactic signaling arrays is cell type specific and not a consequence of swarm cell elongation. Intracellular localization of YFP-CheW in wild-type swimmer cells of $V$. parahaemolyticus treated with the antibiotic Aztreonam in order to artificially induce elongation of swimmer cells. (A) Microscopy showing the intracellular localization of YFP-CheW. (B) Graph depicting the distance of YFP-CheW foci from the cell poles as a function of cell. (C) Demographic analysis showing the fluorescence intensity of YFP-CheW along the cell length in a population of $\mathrm{V}$. parahaemolyticus cells relative to cell length.

observed, just like seen in swimmer cells grown in liquid medium (Figure 7B). Thus, within swarm-colonies cells not only display morphological differences, but also regulate their intracellular organization and localization of chemotactic signaling arrays depending on their specific position within the colony.

\section{DISCUSSION}

We have performed a detailed analysis of the intracellular localization of chemotactic signaling arrays during the life cycles of $V$. parahaemolyticus and shown that in contrast to swimmer cells, where chemotactic signaling arrays are strictly confined to the cell poles, swarmer cells have two distinct localization patterns; bi-polar localization and lateral localization along the cell length (Gestwicki et al., 2000). Here, we show data suggesting that the formation and localization of polar and lateral arrays in swarmer cells are driven by distinct mechanisms. Additionally, we show that there is a correlation between swarmer cell length and the number of signaling arrays formed within the swarmer cell; long swarmer cells possess a higher number of signaling arrays compared to short swarmer cells and the number of lateral clusters formed increases with increased cell length. We show that lateral signaling arrays are localized in a non-regular manner but on a population average each cell halve hold the same amount of lateral signaling arrays. This distribution possibly ensures that upon cell division each daughter swarmer cell is likely to inherit laterally localized signaling arrays.

Using immunofluorescence on fixed cells it has previously been reported that lateral signaling arrays form in elongated cells of $V$. parahaemolyticus from liquid medium (Gestwicki et al., 2000). These experiments were performed in cells deleted for
lonS that have partially initiated the swarmer cell differentiation program and are elongated in liquid media; the exact role of the protease LonS in regulation of differentiation, however, is not known, and pleiotropic effects cannot be excluded (Stewart et al., 1997). Here, we analyzed the localization in live genetically defined cells, both in bona fide swarmers and in artificially elongated swimmer cells from liquid medium that have not initiated the swarm program. We observe no formation of the non-regularly distributed arrays along the length of the artificially elongated swimmer cells, while the bona fide swarmers resemble cells lacking lonS in displaying lateral signaling arrays, hence suggesting that formation of nonregularly distributed signaling arrays is specific for cells that have initiated swarmer differentiation and not simply a consequence of cell elongation.

Interestingly, our data also suggest that swarmer cells employ two distinct mechanisms for localization of signaling arrays: First, the ParC system is responsible for recruitment of signaling arrays to the cell poles and the establishment of a bi-polar localization of signaling arrays. Second, a different mechanism regulates formation and positioning of lateral signaling arrays along the length of swarmer cells. Our data suggests that this mechanism specifically comes into action after entering the differentiation program to swarmer cells and is independent of cell length. It might be similar to the situation in the peritrichously flagellated $E$. coli, where formation of signaling arrays is driven by a stochastic process (Thiem and Sourjik, 2008), resulting in localization of signaling arrays at the cell poles and non-regularly along the cell length (Sourjik and Berg, 2000). V. parahaemolyticus encodes a large number of different chemoreceptors suggesting a pronounced capability for sensing and responding to environmental signals. Thus, one possibility is that one or more of these receptors are specific 


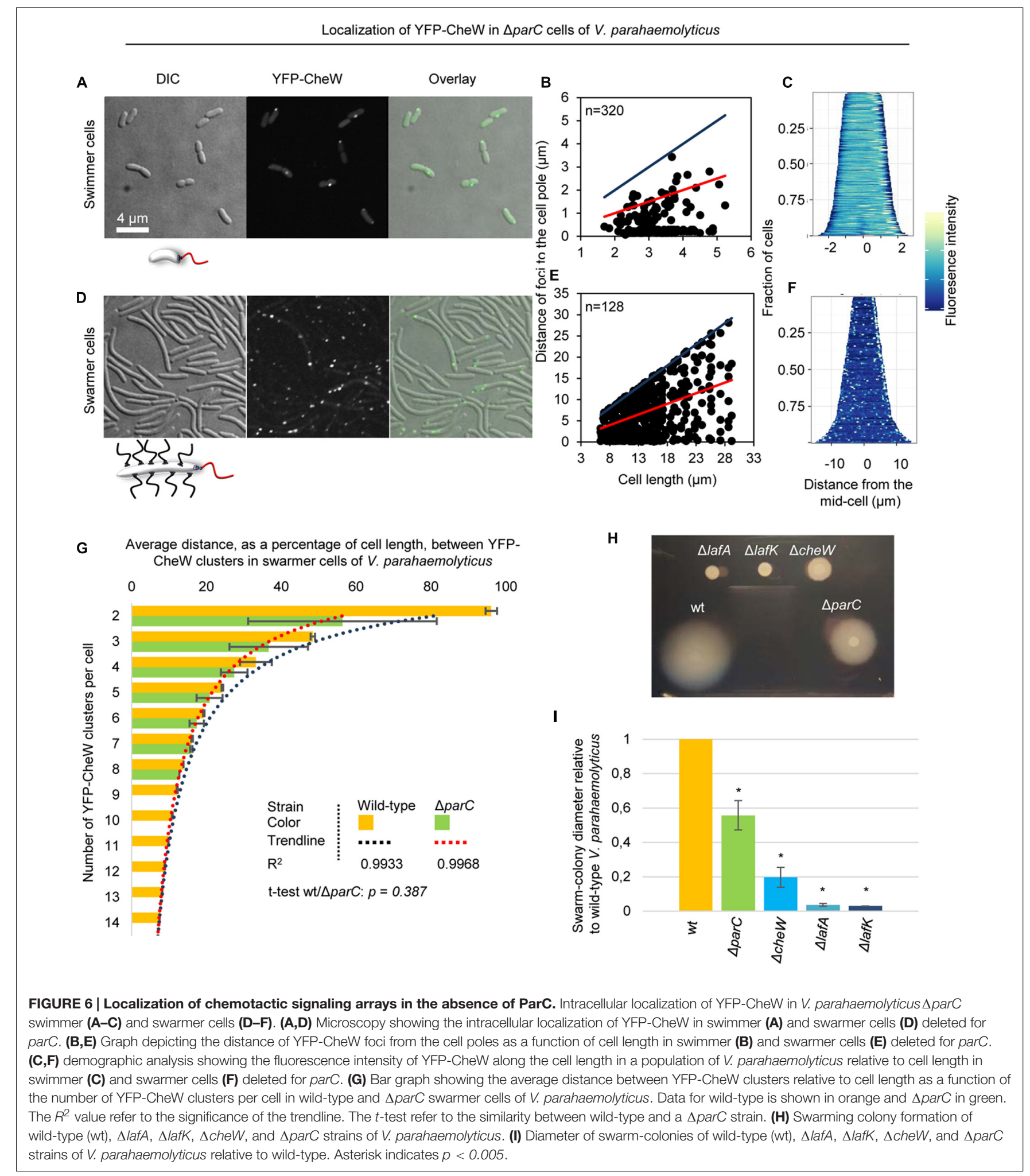

for swarmer cells and play a role in the formation of lateral signaling arrays: the ParC-system might not interact with those swarmer specific receptors, thereby allowing formation of lateral arrays driven by stochastic assembly similar to that observed for E. coli where individual receptors are inserted randomly in the membrane, in which they diffuse freely and either join existing arrays or nucleate new ones (Sourjik and Berg, 2000; Thiem and Sourjik, 2008). This suggestion is supported 

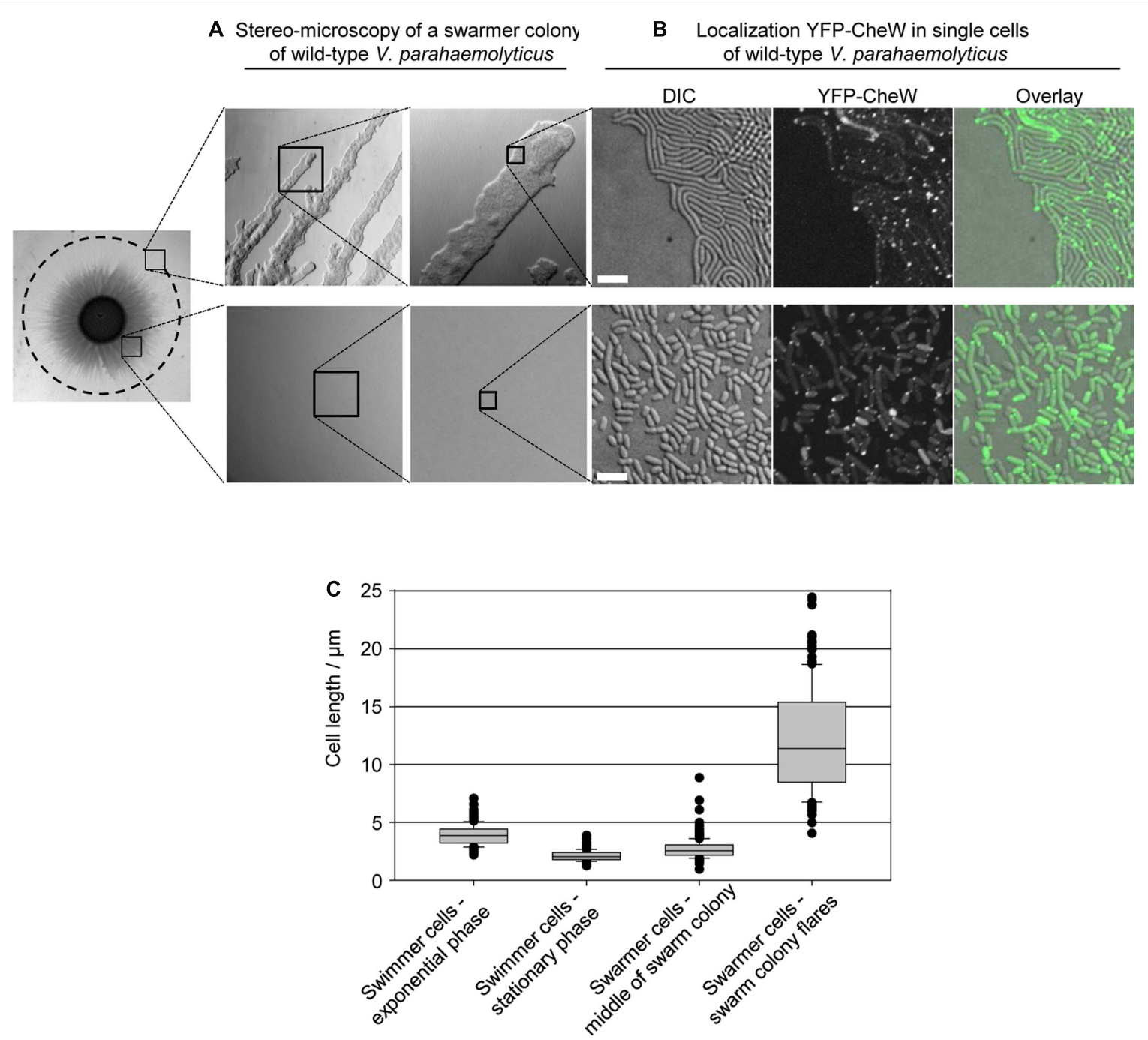

FIGURE 7 | Localization of signaling arrays in swarmer cells changes with swarm-colony development. (A) Stereo-microscopy of a swarmer colony of V. parahaemolyticus. Upper panel shows the swarm flares at higher magnifications, and lower panel shows the middle of a swarm colony at higher magnifications. (B) Fluorescence microscopy showing the localization of YFP-CheW in V. parahaemolyticus cells from the periphery (swarm flares) and middle of swarm colonies. Images are representative of areas indicated in (A). Cells from the periphery were imprinted on microscope slides. Due to cells being stacked in multilayers, cells from the middle were scraped off plates, re-suspended in medium and spotted on a microscope slide, in order to obtain single layered cells for microscopy. (C) Graph showing the cell-length distribution of $V$. parahaemolyticus depending on its growth phase and position within swarm colonies; swimmer cells - exponential phase, $n=297$; swimmer cells stationary phase, $n=300$; swarmer cell - middle, $n=307$; swarmer cells - periphery, $n=151$.

by microarray comparison between surface and liquid grown $V$. parahaemolyticus, where both up- and down-regulation in expression of several MCPs and predicted chemotaxis proteins, depending on the differentiation state, was detected (GodePotratz et al., 2011). Alternatively, the formation of lateral chemosensory arrays may be driven by a component of the lateral flagellar systems, which - similarly to the lateral chemotaxis arrays - are located in a non-regular manner along the length of the cell. It is likely that the formation and non-uniform distribution of signaling arrays along the cell length in swarmer cells ensures that chemotaxis sensory arrays are localized in close proximity to the randomly localized flagella along the cell body.
Interestingly, the actual role of chemotaxis during swarming is still not fully understood. It is known that in E. coli and Salmonella the chemotaxis system is required for swarming, however, there is also evidence indicating that it might not be chemotaxis per se that is required but instead the chemotaxis system plays a mechanical role in swarming motility (Burkart et al., 1998; Mariconda et al., 2006). Furthermore, it has been suggested that in E. coli interactions between cell bodies are responsible for swarm colony expansion and cell reversals rather than the chemotaxis system itself (Damton et al., 2010). In $V$. parahaemolyticus transposon insertion mutants have been isolated that simultaneously result both in chemotaxis and swarming defects, and experiments suggested that the chemotaxis 
system influences both the polar and lateral flagella systems (Sar et al., 1990; McCarter, 2004). The mutations map to two regions on chromosome 1 near the polar flagellar gene clusters, and likely insert in the chemotaxis gene operon. The exact insertion sites, however, have not been identified. Here, we used a clean genetic construct specifically deleted for the chemotaxis protein CheW, responsible for chemotactic behavior of swimmer cells (Ringgaard et al., 2014), and show that the swarming behavior of $V$. parahaemolyticus is clearly affected. Nonetheless, the actual mechanism by which chemotaxis influences swarming remains to be elucidated.

Our data also show that there are differences in the subcellular localization patterns of chemosensory arrays depending on the cell's position within a swarm colony. It is possible that, as elongated swarmer cells spread over surfaces, cells that remain in the middle of the swarm colony dedifferentiate back into swimmers cells, and thus only position their signaling arrays at the cell pole in proximity of the polar flagellum required for swimming behavior. The natural habitat of $V$. parahaemolyticus is the marine environment, therefore it may be important to maintain a constant population of swimmers at all times. Thus, separating into two distinct cell populations within swarm colonies likely allows for swarming across surfaces while maintaining a continuous

\section{REFERENCES}

Alberti, L., and Harshey, R. M. (1990). Differentiation of Serratia marcescens 274 into swimmer and swarmer cells. J. Bacteriol. 172, 4322-4328.

Alley, M. R., Maddock, J. R., and Shapiro, L. (1992). Polar localization of a bacterial chemoreceptor. Genes Dev. 6, 825-836. doi: 10.1101/gad.6.5.825

Bardy, S. L., and Maddock, J. R. (2005). Polar localization of a soluble methylaccepting protein of Pseudomonas aeruginosa. J. Bacteriol. 187, 7840-7844. doi: 10.1128/JB.187.22.7840

Baumann, P., and Baumann, L. (1977). Biology of the marine enterobacteria: genera beneckea and Photobacterium. Annu. Rev. Microbiol. 31, 39-61. doi: 10.1146/annurev.mi.31.100177.000351

Belas, M. R., and Colwell, R. R. (1982). Scanning electron microscope observation of the swarming phenomenon of Vibrio parahaemolyticus. J. Bacteriol. 150, 956-959.

Böttcher, T., Elliott, H. L., and Clardy, J. (2016). Dynamics of snake-like swarming behavior of Vibrio alginolyticus. Biophys. J. 110, 981-992. doi: 10.1016/j.bpj.2015.12.037

Briegel, A., Ortega, D. R., Mann, P., Kjær, A., Ringgaard, S., and Jensen, G. J. (2016). Chemotaxis cluster 1 proteins form cytoplasmic arrays in Vibrio cholerae and are stabilized by a double signaling domain receptor DosM. Proc. Natl. Acad. Sci. U.S.A. 113, 10412-10417. doi: 10.1073/pnas.1604693113

Burkart, M., Toguchi, A., and Harshey, R. M. (1998). The chemotaxis system, but not chemotaxis, is essential for swarming motility in Escherichia coli. Proc. Natl. Acad. Sci. U.S.A. 95, 2568-2573. doi: 10.1073/pnas.95.5.2568

Damton, N. C., Turner, L., Rojevsky, S., and Berg, H. C. (2010). Dynamics of bacterial swarming. Biophys. J. 98, 2082-2090. doi: 10.1016/j.bpj.2010.01.053

Gestwicki, J. E., Lamanna, A. C., Harshey, R. M., Mccarter, L. L., Kiessling, L. L., and Adler, J. (2000). Evolutionary conservation of methyl-accepting chemotaxis protein location in bacteria and archaea evolutionary conservation of methylaccepting chemotaxis protein location in bacteria and archaea. J. Bacteriol. 182, 6499-6502. doi: 10.1128/JB.182.22.6499-6502.2000.Updated

Gode-Potratz, C. J., Kustusch, R. J., Breheny, P. J., Weiss, D. S., and McCarter, L. L. (2011). Surface sensing in Vibrio parahaemolyticus triggers a programme of gene expression that promotes colonization and virulence. Mol. Microbiol. 79, 240-263. doi: 10.1111/j.1365-2958.2010.07445.x pool of swimmer cells that are ready to be released into liquid environments and immediately capable of exploring new surroundings.

\section{AUTHOR CONTRIBUTIONS}

JH carried out the experimental work, participated in data analysis, participated in the design of the study, and helped drafting the manuscript. SR masterminded the work, participated in data analysis, participated in the design of the study, and drafted the manuscript.

\section{FUNDING}

This work was supported by the Max Planck Society.

\section{ACKNOWLEDGMENTS}

We are grateful to Dr. Kathrin Schirner for comments on the manuscript and for suggestions for experiments. We thank Prof. Dr. Lotte Søgaard-Andersen for comments on the manuscript.

Gode-Potratz, C. J., and McCarter, L. L. (2011). Quorum sensing and silencing in Vibrio parahaemolyticus. J. Bacteriol. 193, 4224-4237. doi: 10.1128/JB.00432-11

Harshey, R. M. (1994). Bees aren't the only ones: swarming in gram-negative bacteria. Mol. Microbiol. 13, 389-394. doi: 10.1111/j.1365-2958.1994.tb00433.x

Harshey, R. M., and Matsuyama, T. (1994). Dimorphic transition in Escherichia coli and Salmonella typhimurium: surface-induced differentiation into hyperflagellate swarmer cells. Proc. Natl. Acad. Sci. U.S.A. 91, 8631-8635. doi: 10.1073/pnas.91.18.8631

Kirov, S. M., Tassell, B. C., Semmler, A. B. T., Donovan, L. A. O., Rabaan, A. A., and Shaw, J. G. (2002). Lateral flagella and swarming motility in Aeromonas Species. J. Bacteriol. 184, 547-555. doi: 10.1128/JB.184.2.547

Maddock, J. R., and Shapiro, L. (1993). Polar localization of the chemotreceptor complex in Escherichia coli cell. Science 259, 1717-1723. doi: 10.1126/science.8456299

Makino, K., Oshima, K., Kurokawa, K., and Yokoyama, K. (2003). Genome sequence of Vibrio parahaemolyticus: a pathogenic mechanism distinct from that of V. cholerae. Lancet 361, 743-749. doi: 10.1016/S0140-6736(03) 12659-1

Mariconda, S., Wang, Q., and Harshey, R. M. (2006). A mechanical role for the chemotaxis system in swarming motility. Mol. Microbiol. 60, 1590-1602. doi: 10.1111/j.1365-2958.2006.05208.x

McCarter, L. (1999). The multiple identities of Vibrio parahaemolyticus. J. Mol. Microbiol. Biotechnol. 1, 51-57.

McCarter, L., and Silverman, M. (1990). Surface-induced swarmer cell differentiation of Vibrio parahaemolyticus. Mol. Microbiol. 4, 1057-1062. doi: 10.1111/j.1365-2958.1990.tb00678.x

McCarter, L. L. (2004). Dual flagellar systems enable motility under different circumstances. J. Mol. Microbiol. Biotechnol. 7, 18-29. doi: 10.1159/000077866

McCarter, L. L. (2010). Bacterial acrobatics on a surface: swirling packs, collisions, and reversals during swarming. J. Bacteriol. 192, 3246-3248. doi: 10.1128/JB.00434-10

Milton, D. L., O’Toole, R., Horstedt, P., and Wolf-Watz, H. (1996). Flagellin a is essential for the virulence of Vibrio anguillarum. J. Bacteriol. 178, 1310-1319.

R Development Core Team (2008). R: A Language and Environment for Statistical Computing. $R$ Found. Stat. Comput. Vienna: R Foundation for Statistical Computing. 
Rather, P. N. (2005). Swarmer cell differentiation in Proteus mirabilis. Environ. Microbiol. 7, 1065-1073. doi: 10.1111/j.1462-2920.2005.00806.x

Ringgaard, S., Hubbard, T., Mandlik, A., Davis, B. M., and Waldor, M. K. (2015). RpoS and quorum sensing control expression and polar localization of Vibrio cholerae chemotaxis cluster III proteins in vitro and in vivo. Mol. Microbiol. 97, 660-675. doi: 10.1111/mmi.13053

Ringgaard, S., Schirner, K., Davis, B. M., and Waldor, M. K. (2011). A family of ParA-like ATPases promotes cell pole maturation by facilitating polar localization of chemotaxis proteins. Genes Dev. 25, 1544-1555. doi: 10.1101/gad.2061811.and

Ringgaard, S., Zepeda-Rivera, M., Wu, X., Schirner, K., Davis, B. M., and Waldor, M. K. (2014). ParP prevents dissociation of CheA from chemotactic signaling arrays and tethers them to a polar anchor. Proc. Natl. Acad. Sci. U.S.A. 111, E255-E264. doi: 10.1073/pnas.1315722111

Roth, D., Finkelshtein, A., Ingham, C., Helman, Y., Sirota-Madi, A., Brodsky, L., et al. (2013). Identification and characterization of a highly motile and antibiotic refractory subpopulation involved in the expansion of swarming colonies of Paenibacillus vortex. Environ. Microbiol. 15, 2532-2544. doi: 10.1111/14622920.12160

Sar, N., McCarter, L., Simon, M., and Silverman, M. (1990). Chemotactic control of the two flagellar systems of Vibrio parahaemolyticus. J. Bacteriol. 172, 334-341.

Sourjik, V., and Armitage, J. P. (2010). Spatial organization in bacterial chemotaxis. EMBO J. 29, 2724-2733. doi: 10.1038/emboj.2010.178

Sourjik, V., and Berg, H. C. (2000). Localization of components of the chemotaxis machinery of Escherichia coli using fluorescent protein fusions. Mol. Microbiol. 37, 740-751. doi: 10.1046/j.1365-2958.2000.02044.x

Stewart, B., and McCarter, L. (2003). Lateral flagellar gene system of Vibrio parahaemolyticus. J. Bacteriol. 185, doi: 10.1128/JB.185.15.4508
Stewart, B. J., Enos-Berlage, J. L., and McCarter, L. L. (1997). The lonS gene regulates swarmer cell differentiation of Vibrio parahaemolyticus. J. Bacteriol. $179,107-114$.

Thiem, S., and Sourjik, V. (2008). Stochastic assembly of chemoreceptor clusters in Escherichia coli. Mol. Microbiol. 68, 1228-1236. doi: 10.1111/j.13652958.2008.06227.x

Wadhams, G. H., and Armitage, J. P. (2004). Making sense of it all: bacterial chemotaxis. Nat. Rev. Mol. Cell Biol. 5, 1024-1037. doi: 10.1038/nrm1524

Wadhams, G. H., Warren, A. V., Martin, A. C., and Armitage, J. P. (2003). Targeting of two signal transduction pathways to different regions of the bacterial cell. Mol. Microbiol. 50, 763-770. doi: 10.1046/j.1365-2958.2003.03716.x

Wickham, H. (2009). Hadley Wickham. Media 35:211. doi: 10.1007/978-0-38798141-3

Yamaichi, Y., Bruckner, R., Ringgaard, S., Cameron, D. E., Briegel, A., Jensen, G. J., et al. (2012). A multidomain hub anchors the chromosome segregation and chemotactic machinery to the bacterial pole. Genes Dev. 26, 2348-2360. doi: 10.1101/gad.199869.112

Conflict of Interest Statement: The authors declare that the research was conducted in the absence of any commercial or financial relationships that could be construed as a potential conflict of interest.

Copyright $\odot 2016$ Heering and Ringgaard. This is an open-access article distributed under the terms of the Creative Commons Attribution License (CC BY). The use, distribution or reproduction in other forums is permitted, provided the original author(s) or licensor are credited and that the original publication in this journal is cited, in accordance with accepted academic practice. No use, distribution or reproduction is permitted which does not comply with these terms. 